\title{
COUNTING 2-CIRCULANT GRAPHS
}

\author{
GEK-LING CHIA and CHONG-KEANG LIM
}

(Received 15 October 1983)

Communicated by W. D. Wallis

\begin{abstract}
Alspach and Sutcliffe call a graph $X(S, q, F)$ 2-circulant if it consists of two isomorphic copies of circulant graphs $X(p, S)$ and $X(p, q S)$ on $p$ vertices with "cross-edges" joining one another in a prescribed manner. In this paper, we enumerate the nonisomorphic classes of 2-circulant graphs $X(S, q, F)$ such that $|S|=m$ and $|F|=k$. We also determine a necessary and sufficient condition for a 2-circulant graph to be a GRR. The nonisomorphic classes of GRR on $2 p$ vertices are also enumerated.
\end{abstract}

1980 Mathematics subject classification (Amer. Math. Soc.): 05 C 25, 05 C 30, 05 C 99.

\section{Introduction}

We consider only finite undirected graphs with no loops or multiple edges. Definitions not given here may be found in [10]. Let $Z_{n}$ be the ring of integers and $Z_{n}^{*}$ the multiplicative group of units in $Z_{n}$. Let $S$ be a subset of $Z_{n}^{*}$ with $S=-S$. The circulant graph $X=X(n, S)$ with symbol $S$ is the graph with vertex set $u_{0}$, $u_{1}, \ldots, u_{n-1}$ and an edge joining $u_{i}$ and $u_{j}$ if and only if $j-i \in S$. Let $p$ denote a prime number. Turner [12] shows that two circulant graphs $X(p, S)$ and $X\left(p, S^{\prime}\right)$ are isomorphic if and only if $S^{\prime}=q S$ for some $q$ in $Z_{p}^{*}$. He also gave an enumerative polynomial for this class of circulant graphs. The automorphism group $A(X)$ of a circulant graph $X=X(p, S)$ was determined explicitly by Alspach [1]. If $\varnothing \subset S \subseteq Z_{p}^{*}$, then $A(X)$ has order $|E(S)| p$ where $E(S)$ is the largest subgroup of $Z_{p}^{*}$ for which $S$ can be written as a union of cosets of $E(S)$. If $\beta^{*}(p, m, d)$ denotes the number of nonisomorphic circulant graphs $X=X(p, S)$

(C) 1985 Australian Mathematical Society $0263-6115 / 85 \$ A 2.00+0.00$ 
with degree $|S|=m$ and automorphism group of order $d p$, then

$$
\beta^{*}(p, m, d)=\frac{d}{m} \sum_{d \mid d_{i}} \mu\left(\frac{d_{i}}{d}\right)\left(\begin{array}{c}
\frac{p-1}{d_{i}}-1 \\
\frac{m}{d_{i}}-1
\end{array}\right)
$$

where $\mu(n)$ is the classical Möbius function (see [5] Chapter 5).

In [2] Alspach and Sutcliffe call a graph $X=X(S, q, F)$ 2-circulant if (i) $V(X)=V\left(X_{1}\right) \cup V\left(X_{2}\right)$ where $X_{1}=X(p, S)$ and $X_{2}=X(p, q S)$ are two isomorphic circulant graphs with $V\left(X_{j}\right)=\left\{u_{j, 0}, u_{j, 1}, \ldots, u_{j, p-1}\right\}, j=1$, 2, (ii) $E(X)$ $=E\left(X_{1}\right) \cup E\left(X_{2}\right) \cup\left\{\left(u_{1, i}, u_{2, j}\right) \mid j-i \in F\right\}$, (iii) $q$ is chosen such that $q^{2} \in$ $E(S)$ and (iv) (a) if $q S=S$ then $F$ is any subset of $Z_{p}$, (b) if $q S \neq S$ then $j \in F$ implies that $-q j \in F$. A 2-circulant $X(S, q, F)$ is said to be of Type-I if it has a representation $X\left(S^{\prime}, q^{\prime}, F^{\prime}\right) \cong X(S, q, F)$ with $q^{\prime}=1$; otherwise it is said to be of Type-II. In this paper we enumerate separately, the nonisomorphic classes of Type-I and Type-II 2-circulants such that $|S|=m$ and $|F|=k$. Our method is similar to the one used in [4].

It is not difficult to see that a GRR on $2 p$ vertices is a Type-I 2-circulant. In the final section, we determine a necessary and sufficient condition for a Type-I 2-circulant graph to be a GRR (Theorem 4.3). We then proceed to enumerate the nonisomorphic classes of GRR on $2 p$ vertices.

\section{Type-I 2-circulants}

In this section we shall count the number of Type-I 2-circulant graphs. Theorem 8 of [2] asserts that $X(S, q, F)$ with $|F| \neq 0$ is of Type-I if and only if $q S=S$. Now this is possible if and only if $q \in E(S)$. So we may assume without loss of generality that $q=1$ whenever $X(S, q, F)$ is Type-I.

Let $2 I(p, m, k)$ denote the number of nonisomorphic Type-I 2-circulant graphs $X(S, 1, F)$ with $|S|=m$ (even) and $|F|=k$. We note that $2 I(p, m, k)=$ $2 I(p, m, p-k)=2 I(p, p-m-1, p-k)$ for any $k \geqslant 0$ and $m>0$. We note further that if $k=0$ or $k=1$, then $2 I(p, m, k)$ is the number of circulant graphs $X(p, S)$ with $|S|=m$. In view of these we may assume that $2 \leqslant|F| \leqslant$ $(p-1) / 2$ and $0<m \leqslant(p-1) / 2$.

THEOREM 2.1 [2, Theorem 10]. Two Type-I 2-circulant graphs $X(S, 1, F)$ and $X\left(S, 1, F^{\prime}\right)$ with $\varnothing \subset F, F^{\prime} \subset Z_{p}$ are isomorphic if and only if there exists $\alpha \in E(S)$ such that $F^{\prime}=\alpha F+c$ for some $c \in Z_{p}$. 
Let $X=X(S, 1, F)$ and $\mathscr{F}(k)$ be the collection of all subsets of $Z_{p}$ each of cardinality $k>0$. Suppose $k<p$ and let $F \in \mathscr{F}(k)$. By Theorem 2.1, $X(S, 1, F+c) \cong X(S, 1, F+d)$ for any $c, d \in Z_{p}$. Now since $F+c \neq F+d$ if $c \neq d$, each $F \in \mathscr{F}(k)$ induces a family containing $p$ elements and so there are $n=(1 / p)\left(\begin{array}{l}p \\ k\end{array}\right)$ families in $\mathscr{F}(k)$. Let these families be $\mathscr{F}_{1}, \ldots, \mathscr{F}_{n}$. Two families $\mathscr{F}_{i}$ and $\mathscr{F}_{j}$ are said to be equivalent under $E(S)$, written $\mathscr{F}_{i} \sim \mathscr{F}_{j}$ if there exist $\alpha \in E(S), F \in \mathscr{F}_{i}$ and $F^{\prime} \in \mathscr{F}_{j}$ such that $\alpha F=F^{\prime}+c$ for some $c \in Z_{p}$. Evidently $\sim$ is an equivalence relation and that if $\mathscr{F}_{i} \sim \mathscr{F}_{j}$, then $X(S, 1, F) \cong$ $X\left(S, 1, F^{\prime}\right)$ for any $F \in \mathscr{F}_{i}$ and $F^{\prime} \in \mathscr{F}_{j}$. By Theorem 2.1 the action of $E(S)$ will partition $\mathscr{F}_{1}, \ldots, \mathscr{F}_{n}$ into equivalence classes. We shall determine the number of these equivalence classes.

Let $E_{i}$ be the subgroup of $Z_{p}^{*}$ with $\left|E_{i}\right|=d_{i}$. Then $\mathscr{F}$ is said to be invariant under $E_{i}$, if for every $F \in \mathscr{F}$, we have $\alpha F=F+c$ for any $\alpha \in E_{i}$ and some $c \in Z_{p}$.

LEMMA 2.2. F is invariant under $E_{i} \neq 1$ if and only if there exists $F \in \mathscr{F}_{\text {such }}$ that $F=\bigcup_{\alpha} \alpha E_{i}$ or $F \backslash\{0\}=\bigcup_{\alpha} \alpha E_{i}$.

Proof. The sufficiency is clear. If $\mathscr{F}$ is invariant under $E_{i}$, then for every $F \in \mathscr{F}$, we have $a F=F+c$ for some $c \in Z_{p}$ and any $a \in E_{i}$. In particular we choose $F$ such that $c=0$. Since $a F=F$ if and only if $a \in E_{i}$, it follows that either $F=\bigcup_{\alpha} \alpha E_{i}$ or $F \backslash\{0\}=\bigcup_{\alpha} \alpha E_{i}$.

LEMma 2.3. Let $A_{1}=\bigcup_{\alpha} \alpha E_{i}, A_{2}=\cup_{\beta} \beta E_{i}$ where $E_{i} \neq 1$. If $A_{1} \neq A_{2}$, then $A_{1} \neq A_{2}+c$ for any $c \in Z_{p}$.

Proof. We need only note that for $E_{i} \neq 1$ and $j=1,2, \sum_{r \in A_{j}} r \equiv 0(\bmod p)$ and that $c\left|A_{2}\right| \equiv 0(\bmod p)$ unless $c=0$ or $\left|A_{2}\right|=p$. But these are ruled out by the fact that $A_{1} \neq A_{2}$.

Combining Lemmas 2.2 and 2.3, we have

LEMMA 2.4. The number of families which are invariant under some non-trivial subgroup $E_{i}$ of $E(S)$ is given by

$$
\Psi\left(E_{i} ; F\right)=\left\{\begin{array}{cl}
\left(\begin{array}{c}
(p-1) / d_{i} \\
|F| / d_{i}
\end{array}\right) & \text { if } d_{i} \text { divides }|F|, \\
\left(\begin{array}{c}
(p-1) / d_{i} \\
(|F|-1) / d_{i}
\end{array}\right) & \text { if } d_{i} \text { divides }|F|-1, \\
0 & \text { otherwise. }
\end{array}\right.
$$


Since the identity subgroup $E_{0}=1$ of $E(S)$ leaves all families invariant, $\Psi(1 ; F)=(1 / p)\left({ }_{|F|}^{p}\right)$.

For each $i \geqslant 0$ and $d_{i}$ dividing $d=|E(S)|$, let $\mathscr{A}_{i}$ denote the collection of all $\mathscr{F}$ which are invariant under $E_{i} \leqslant E(S)$. Then $\left|\mathscr{A}_{i}\right|=\Psi\left(E_{i} ; F\right)$.

LEMMA 2.5. $\mathscr{A}_{i} \cap \mathscr{A}_{j}=\mathscr{A}_{j}$ if and only if $d_{i}$ divides $d_{j}$.

Proof. Now $d_{i}$ divides $d_{j}$ implies that $E_{i} \leqslant E_{j}$ so that any $\mathscr{F}$ invariant under $E_{j}$ is also invariant under $E_{i}$. Now if $\mathscr{A}_{i}=\mathscr{A}_{j}$, the result is true. So we may assume that $\mathscr{A}_{j} \subset \mathscr{A}_{i}$ and that there is no $\mathscr{A}_{k}$ with $\mathscr{A}_{j} \subset \mathscr{A}_{k} \subset \mathscr{A}_{i}$. Let $\mathscr{F} \in \mathscr{A}_{j}$. By Lemma 2.2 , there exists $F \in \mathscr{F}$ such that either $F=\bigcup_{\alpha} \alpha E_{j}$ or $F \backslash\{0\}=\bigcup_{\alpha} \alpha E_{j}$. Since $\mathscr{A}_{j} \subset \mathscr{A}_{i}, \mathscr{F}$ is also invariant under $E_{i}$, and the same $F \in \mathscr{F}$ will have the property that $a F=F$ for all $a \in E_{i}$. Hence either $F=\bigcup_{\alpha} \alpha E_{i}$ or $F \backslash\{0\}=\bigcup_{\alpha} \alpha E_{i}$. Thus we have either $F=\bigcup_{\alpha} \alpha E_{j}=\bigcup_{\alpha} \alpha E_{i}$ or $F \backslash\{0\}=\bigcup_{\alpha} \alpha E_{j}=\bigcup_{\alpha} \alpha E_{i}$. By letting $b \in E_{j} \backslash E_{i}$, we check that $d_{i}$ divides $d_{j}$.

Let $[a, b]$ denote the least common multiple of $a$ and $b$. Using Lemma 2.5, we obtain the following lemma.

LEMMA 2.6. $\mathscr{A}_{i} \cap \mathscr{A}_{j}=\mathscr{A}_{k}$ if and only if $d_{k}=\left[d_{i}, d_{j}\right]$.

LEMma 2.7. $E(S)$ partitions $\mathscr{A}_{i} \backslash \cup_{j} \mathscr{A}_{j}$ into equivalence classes each containing exactly $d / d_{i}$ families.

Proof. Since $E_{i}$ is a subgroup of $E(S)$, we have

$$
E(S)=E_{\imath} \cup g^{k} E_{i} \cup \cdots \cup g^{(n-1) k} E_{i}
$$

where $g$ is a primitive root of $p$ and $k=(p-1) / d$. Since $\mathscr{F} \in \mathscr{A}_{i} \backslash \bigcup_{j} \mathscr{A}_{j}$ is invariant under $E_{i}$, there exists $F \in \mathscr{F}$ with $F=\bigcup_{\alpha} \alpha E_{i}$ or $F \backslash\{0\}=\bigcup_{\alpha} \alpha E_{i}$. Hence the action of $E(S)$ on $\mathscr{A}_{i} \backslash \cup_{j} \mathscr{A}_{j}$ is equivalent to the action of $\left\{1, g^{k}, \ldots, g^{(n-1) k}\right\}$ on $\mathscr{A}_{i} \backslash \cup, \mathscr{A}_{j}$. Now $g^{a k} F \neq g^{b k} F$ whenever $0 \leqslant a \neq b \leqslant n-$ 1. Furthermore we assert that $g^{a k} F \neq g^{b k} F+c$ for any $c \in z_{p}$. This assertion is true for $E_{i} \neq 1$ as can be seen from Lemma 2.3. It remains to show that it is true for $E_{i}=1$. Without loss of generality we show that $F \neq g^{a k} F+c$ for every $c \in Z_{p}$ and $a \neq 0$. Suppose the contrary; then $F=g^{a k} F+c$ for some $c \in Z_{p}$. But this implies that $F=g^{-a k} F+d$ for some $d \in Z_{p}$, and $\mathscr{F}$ is invariant under $\left\{1, g^{a k}, g^{-a k}\right\}$ which is a subgroup of $E(S)$. This contradicts $\mathscr{F} \in \mathscr{A}_{0} \backslash \bigcup_{j} \mathscr{A}_{j}$.

Note that the above lemma does not hold if $|F| \leqslant 1$, or $|F| \geqslant p-1$. However we have assumed earlier that $2 \leqslant|F| \leqslant(p-1) / 2$. Let $2 I^{*}\left(p, d, d_{i},|F|\right)$ denote 
the number of equivalence classes in $\mathscr{A}_{i} \backslash \cup, \mathscr{A}_{j}$. Then by Lemma 2.7 , we have

$$
\frac{d}{d_{i}} 2 I^{*}\left(p, d, d_{i},|F|\right)=\left|\mathscr{A}_{j}\right|-\left|\bigcup_{j} \mathscr{A}_{j}\right| \text {. }
$$

Using Lemma 2.6 and applying the principle of inclusion and exclusion, we obtain

$$
2 I^{*}\left(p, d, d_{i},|F|\right)=\frac{d_{i}}{d} \sum_{d_{i} \mid d_{j}} \mu\left(\frac{d_{j}}{d_{i}}\right) \Psi\left(E_{j} ; F\right) .
$$

Summing up $2 I^{*}\left(p, d, d_{i},|F|\right)$ for all $d_{i}$ which divides $d$, we obtain

$$
2 I^{*}(p, d,|F|)=\sum_{d_{i} \mid d} 2 I^{*}\left(p, d, d_{i},|F|\right)
$$

which is the number of equivalence classes in $\mathscr{F}(k)$ for a fixed $S$ with $|E(S)|=d$. But there are altogether $\beta^{*}(p, m, d)$ non-equivalence $S$ with $|E(S)|=d, 0<|S|$ $\leqslant(p-1) / 2$. Thus summing up $2 I^{*}(p, d,|F|) \beta^{*}(p, m, d)$ for all $d$ even (recall that $d$ is a common divisor of $m$ and $p-1)$, we get

$$
2 I(p, m,|F|)=\sum_{d \text { even }} 2 I^{*}(p, d,|F|) \beta^{*}(p, m, d)
$$

THEOREM 2.8. The number of Type-I 2-circulant graphs $X=X(S, 1, F)$ with $|S|=m$ and $2 \leqslant|F| \leqslant(p-1) / 2$ is given by

$$
2 I(p, m,|F|)=\sum_{d} 2 I^{*}(p, d,|F|) \beta^{*}(p, m, d)
$$

where the summation ranges over all even common divisors $d$ of $m$ and $p-1$.

ExAMPLE 1. Let $p=13, m=6$ and $k=6$. Then the common divisors $d$ of $m$ and $p-1$ are $1<2<3<6$.

(i) When $d=6$,

$$
\begin{aligned}
& 2 I^{*}(p, 6,1,6)=\frac{1}{6} \sum_{1 \mid d_{j}} \mu\left(d_{j}\right) \Psi\left(E_{j} ; F\right)=18, \\
& 2 I^{*}(p, 6,2,6)=\frac{2}{6} \sum_{2 \mid d_{j}} \mu\left(\frac{d_{j}}{2}\right) \Psi\left(E_{j} ; F\right)=6, \\
& 2 I^{*}(p, 6,3,6)=\frac{3}{6} \sum_{3 \mid d_{j}} \mu\left(\frac{d_{j}}{3}\right) \Psi\left(E_{j} ; F\right)=2, \\
& 2 I^{*}(p, 6,6,6)=\frac{6}{6} \sum_{6 \mid d_{j}} \mu\left(\frac{d_{j}}{6}\right) \Psi\left(E_{j} ; F\right)=2 .
\end{aligned}
$$


Thus

$$
\begin{aligned}
& 2 I^{*}(p, 6,6)=\sum_{d \mid 6} 2 I^{*}(p, 6, d, 6)=28 \\
& \beta^{*}(p, m, 6)=\frac{6}{m} \sum_{6 \mid d_{j}} \mu\left(\frac{d_{j}}{6}\right)\left(\begin{array}{c}
\frac{p-1}{d_{j}}-1 \\
\frac{m}{d_{j}}-1
\end{array}\right)=1 .
\end{aligned}
$$

(ii) When $d=2$,

$$
\begin{aligned}
2 I^{*}(p, 2,1,6) & =\frac{1}{2} \sum_{1 \mid d_{j}} \mu\left(d_{j}\right) \Psi\left(E_{j} ; F\right)=56, \\
2 I^{*}(p, 2,2,6) & =\frac{2}{2} \sum_{2 \mid d_{j}} \mu\left(\frac{d_{j}}{2}\right) \Psi\left(E_{j} ; F\right)=18, \\
2 I^{*}(p, 2,6) & =74, \\
\beta^{*}(p, m, 2) & =\frac{2}{m} \sum_{2 \mid d_{j}} \mu\left(\frac{d_{j}}{2}\right)\left(\begin{array}{c}
\frac{p-1}{d_{j}}-1 \\
\frac{m}{d_{j}}-1
\end{array}\right)=3 .
\end{aligned}
$$

Hence

$$
2 I(p, m, 6)=\sum_{d} 2 I^{*}(p, d, 6) \beta^{*}(p, m, d)=250
$$

For the case $H=Z_{p}^{*}$, we note that $E(S)=Z_{p}^{*}$ and that the expression $2 I^{*}\left(p, d, d_{i},|F|\right)$ also works for $d=p-1$. Hence $2 I^{*}(p, p-1,|F|)=$ $\sum_{d \mid p-1} 2 I^{*}(p, p-1, d,|F|)$. However there is only one circulant graph $X(p, S)$ with $S=Z_{p}^{*}$. Thus $2 I(p, p-1,|F|)=2 I^{*}(p, p-1,|F|)$ and we have the following formula which counts the number of weak starred polygons of degree $|F|$ on $2 p$ vertices. (See [6] for the definition of weak starred polygons.)

TheOREM 2.9. $2 I(p, 0,|F|)=2 I(p, p-1,|F|)=\sum_{d \mid p-1} 2 I^{*}(p, p-1, d,|F|)$.

\section{Type-II 2-circulants}

In this section we shall count Type-II 2-circulant graphs $X(S, q, F)$ with $|S|=m$ and $|F|=k$. We proceed by determining the conditions on $q$ which make $X(S, q, F)$ Type-II 2-circulant. 
Proposition 3.1. A 2-circulant graph $X=X(S, q, F)$ with $\varnothing \subset F \subset Z_{p}$ is Type-II if and only if $q=g^{k / 2}$ a for some $a \in E(S)$, where $g$ is a primitive root of $p$ and $k=(p-1) /|E(S)|$.

Proof. Now $Z_{p}^{*}=E(S) \cup g E(S) \cup \cdots \cup g^{k-1} E(S)$. If $X$ is Type-II 2-circulant, then $q S \neq S$ and so $q \notin E(S)$ and $q=g^{\alpha} a$ for some $a \in E(S)$ and $0<\alpha \leqslant k-1$. Now since $q^{2}=g^{2 \alpha} a^{2} \in E(S)$ we must have $g^{2 \alpha} \in E(S)$. But this is possible only if $2 \alpha \equiv 0(\bmod k)$ or $\alpha=k / 2$.

On the other hand if $q=g^{k / 2} a$, then $q S \neq S$. Since $\varnothing \subset F \subset Z_{p}, X$ is a Type-II 2-circulant graph according to Theorem 8 of [2].

Corollary 3.2 [2, Theorem 9]. If $X=X(S, q, F)$ is a Type-II 2-circulant graph, then $p \equiv 1(\bmod 4)$.

Proof. Since $X$ is Type-II 2-circulant, Proposition 3.1 asserts that $k / 2=$ $(p-1) / 2 d$ is an integer. Since $d$ is even, the result follows.

Corollary 3.3. If $X=X(S, q, F)$ is of Type-II 2-circulant, then $2|E(S)|$ divides $p-1$.

Proof. If $X=X(S, q, F)$ is Type-II 2-circulant, then by Proposition 3.1, $k=(p-1) /|E(S)|$ is an even integer. Hence the result follows.

TheOREM 3.4 [2, Theorem 10]. Two Type-II 2-circulant graphs $X(S, q, F)$ and $X\left(S, q^{\prime}, F^{\prime}\right)$ are isomorphic if and only if there exists $\alpha \in E(S)$ with $\alpha F=F^{\prime}$.

Let $\#(a)$ denote the order of $a \in E(S)$.

LEMmA 3.5. If $X=X(S, q, F)$ is Type-II 2-circulant, then $\#\left(q^{2}\right)$ is even.

ProOF. Since $q=g^{k / 2} a=g^{k / 2} g^{r k}, 0 \leqslant r \leqslant d-1$, we have $q^{2}=g^{(2 r+1) k}$. Since $d$ is even, it follows that $q^{2 n}=g^{n(2 r+1) k}=1$ only if $n$ is even. Hence \#( $\left.q^{2}\right)$ is even.

Lemma 3.6. Let $\#\left(q^{2}\right)=d_{i}$. Then for any $j \in F, j E_{v} \subseteq F$ where $d_{v}=2 d_{i}$.

Proof. It suffices to show that $1 \in F$ implies $E_{v} \subseteq F$. Let $1 \in F$. Then by condition (iv)(b), $-q \in F$. Continuing we have $(-1)^{r} q^{r} \in F$ where $0 \leqslant r \leqslant 2 d_{i}-2$. Then $\left\{1, q^{2}, \ldots, q^{2 d_{i}-2}\right\} \cup-q\left\{1, q^{2}, \ldots, q^{2 d_{i}-2}\right\} \subseteq F$. Since $d_{i}$ is even by Lemma 
3.5 , it follows that $-q\left\{1, q^{2}, \ldots, q^{2 d_{i}-2}\right\}=q\left\{1, q^{2}, \ldots, q^{2 d_{i}-2}\right\}$. Since $\#\left(q^{2}\right)=$ $d_{i}$, we see that $q^{2}=g^{k_{i}}$ and so $q=g^{k_{i} / 2}=g^{k_{i}}$. Thus $\left\{1, q^{2}, \ldots, q^{2 d_{i}-2}\right\} \cup$ $q\left\{1, q^{2}, \ldots, q^{2 d_{i}-2}\right\}=E_{v}$ and the lemma follows.

Thus we see that if $|F| \neq 1$, then $F$ depends on the choice of $q$, and for this reason we may sometimes write $F=F(q)$. Since $d_{v}=2 d_{i}=2 \#\left(q^{2}\right)$ and $F=$ $\cup_{\alpha} \alpha E_{v}$ or $F \backslash\{0\}=\bigcup_{\alpha} \alpha E_{v}$, we have the following corollary.

Corollary 3.7. If $X=X(S, q, F)$ is Type-II 2-circulant, then $\varnothing \subset F \subset Z_{p}$ and $|F| \equiv 0\left(\bmod 2 \#\left(q^{2}\right)\right)$ or $|F| \equiv 1\left(\bmod 2 \#\left(q^{2}\right)\right)$.

Let $\mathscr{B}(|F(q)|)$ denote the collection of all $F(q)$ of a Type-II 2-circulant $X(S, q, F(q))$ with $|F(q)|>1$. Then

$$
|\mathscr{B}(|F(q)|)|=\left\{\begin{array}{cl}
\left(\begin{array}{c}
(p-1) / 2 \#\left(q^{2}\right) \\
|F| / 2 \#\left(q^{2}\right)
\end{array}\right) & \text { if }|F|=0\left(\bmod 2 \#\left(q^{2}\right)\right), \\
\left(\begin{array}{c}
(p-1) / 2 \#\left(q^{2}\right) \\
(|F|-1) / 2 \#\left(q^{2}\right)
\end{array}\right) & \text { if }|F| \equiv 1\left(\bmod 2 \#\left(q^{2}\right)\right), \\
0 & \text { otherwise. }
\end{array}\right.
$$

Let $(a, b)$ denote the greatest common divisor of $a$ and $b$.

LEMMA 3.8. The action of $E(S)$ partitions $\mathscr{B}(|F(q)|)$ into equivalence classes each containing exactly $d /\left(2 \#\left(q^{2}\right), d\right)$ elements.

Proof. Let $2 \#\left(q^{2}\right)=d_{v}=2 d_{i}$, and $\left(2 \#\left(q^{2}\right), d\right)=d_{t}$. Then $E_{v} \cap E(S)=E_{t}$ and

$$
\begin{gathered}
E(S)=E_{t} \cup g^{k} E_{t} \cup \cdots \cup g^{\left(d / d_{t}-1\right) k} E_{t}, \\
E_{v}=E_{t} \cup g^{k_{v}} E_{t} \cup \cdots \cup g^{\left(d_{v} / d_{t}-1\right) k_{v}} E_{t} .
\end{gathered}
$$

Since $F$ or $F \backslash\{0\}=\cup_{\alpha} \alpha E_{v}=\cup_{\beta} \beta E_{t}$, we see that the action of $E(S)$ on $\mathscr{B}(|F(q)|)$ is equivalent to the action of $\left\{1, g^{k}, \ldots, g^{\left(d / d_{t}-1\right) k}\right\}$ on $\mathscr{B}(|F(q)|)$. Furthermore $g^{a k} F \neq g^{b k} F$ for any $0 \leqslant a \neq b \leqslant d / d_{t}-1$ and this proves the lemma.

Let $2 I I^{*}(p, d,|F(q)|)$ denote the number of equivalence classes in $\mathscr{B}(|F(q)|)$ for a fixed $S$ with $|E(S)|=d$. Then by Lemma 3.8

$$
2 I I^{*}(p, d,|F(q)|)=\frac{\left(2 \#\left(q^{2}\right), d\right)}{d}|\mathscr{B}(|F(q)|)|,
$$

$|F(q)|>1$. Furthermore, if $F(q)=\{0\}$, then $E(S)$ fixes $F(q)$ so that $2 I I^{*}(p, d,|F(q)|)=1$. Since there are altogether $\beta^{*}(p, m, d)$ non-equivalent $S$ 
with $|E(S)|=d$ and $|S|=m$, on summing $2 I^{*}(p, d,|F(q)|) \beta^{*}(p, m, d)$ for all common divisors $d$ of $m$ and $p-1$ with $2 d$ dividing $p-1$, we obtain $2 I I(p, m,|F(q)|)$, the number of Type-II 2-circulant graphs $X(S, q, F(q))$ with $|S|=m$.

TheOREM 3.9. The number of Type-II 2-circulant graphs $X=X(S, q, F(q))$ with $|S|=m$ is given by

$$
2 I I(p, m,|F(q)|)=\sum_{d} 2 I^{*}(p, d,|F(q)|) \beta^{*}(p, m, d)
$$

where the summation ranges over all even common divisors $d$ of $m$ and $p-1$ with $2 d$ dividing $p-1$.

Remark. If $S=\varnothing, S=Z_{p}^{*}$ or $F=\varnothing, F=Z_{p}$ then $X(S, q, F)$ is Type-I 2-circulant. Hence $2 I I(p, 0, k)=0=2 I I(p, m, 0)$. Note that $2 I I(p, m,|F(q)|)$ $=2 I I(p, p-1-m, p-|F(q)|)$. Furthermore since $2 I I^{*}(p, d,|F(q)|)=$ $2 I I^{*}(p, d, p-|F(q)|)$ it follows that $2 I I(p, m,|F(q)|)=2 I I(p, m, p-|F(q)|)$.

EXAMPLE 2. Let $p=13, m=6$ and $|F(q)|=4$. Then the even common divisors $d$ of $m$ and $p-1$ are 2 and 6 .

(i) When $d=2, \#\left(q^{2}\right)=2$ and so $2 I I^{*}(p, d, 4)=3$ and $\beta^{*}(p, m, 2)=2$.

(ii) When $d=6$, then either \#( $\left.q^{2}\right)=2$ or $\#\left(q^{2}\right)=6$. Since $2 \#\left(q^{2}\right)$ must divide $|F(q)|$, only $\#\left(q^{2}\right)=2$ is possible. So $2 I I^{*}(p, d, 4)=1$ and $\beta^{*}(p, m, 6)$ $=1$.

Thus $2 I I(p, m,|F(q)|)=\Sigma_{d} 2 I^{*}(p, m, d) \beta^{*}(p, m, d)=10$.

\section{GRR on $2 p$ vertices}

Let $G$ be a finite group and $H$ a subset of $G$ with the properties (i) $1 \notin H$ and (ii) $h \in H$ implies $h^{-1} \in H$. Then the Cayley graph of $G$ with respect to the generating set $H$ is the graph $X_{G, H}$ with $V\left(X_{G, H}\right)=G$ and $E\left(X_{G, H}\right)=\{(g, g h) \mid h$ $\in H\}$. Clearly $X_{G, H}$ is connected if and only if $\langle H\rangle=G$. A graph $X$ is called a graphical regular representation (GRR) of a group $G$ if the automorphism group $A(X)$ of $X$ is regular, as a permutation group, and isomorphic to $G$. Sabidussi in [11] shows that if $X$ is a GRR, then $X \cong \bar{K}_{2}$, or else $X$ is connected and $X \cong X_{G, H}$ for some group $G$ and some generating set $H$ of $G$. In this section we shall apply the method developed in Section 2 to count the number of GRR on $2 p$ vertices. A special case of our result is a partial solution (Corollary 4.7) to a problem (8b) raised in [9]: which groups have a cubic GRR? 
We remark that the set of Type-I 2-circulant graphs coincides with the set of Cayley graphs $X_{D_{p}, J}$. (Here $D_{p}=\left\langle a, b \mid a^{p}=b^{2}=1, b a b=a^{-1}\right\rangle$ denotes the dihedral group of order $2 p$.) For if $X=X_{D_{p}, H}$, then $X$ is of Type-I 2-circulant $X(S, 1, F)$ with $S=\left\{i \mid a^{i} \in H\right\}$ and $F=\left\{i \mid a^{i} b \in H\right\}$. Conversely if $X=$ $X(S, 1, F)$, then $X$ is a Cayley graph on the cyclic group $Z_{2 p}$, or on the dihedral group $D_{p}$ [2, Theorem 6]. Moreover if $X=X_{Z_{2 p}, H}$, then $X \cong X_{D_{p}, H^{\prime}}$ for some generating set $H^{\prime}$ of $D_{p}$.

Let $\alpha \in Z_{p}^{*}$ and $c \in Z_{p}$ and define $\psi_{\alpha, c}: D_{p} \rightarrow D_{p}$ by $\psi_{\alpha, c}\left(a^{i}\right)=a^{\alpha i}$ and $\psi_{\alpha, c}\left(a^{i} b\right)=a^{\alpha i+c} b$. Then the automorphism group of $D_{p}$ is $A\left(D_{p}\right)=\left\{\psi_{\alpha, c} \mid \alpha \in Z_{p}^{*}\right.$, $c \in Z_{p}$ \} [6, Lemma 2]. Now any isomorphism $\psi$ of $X_{1}=X_{D_{p}, H}$ onto $X_{2}=X_{D_{p}, H^{\prime}}$ with $\psi(H)=H^{\prime}$ corresponds to an isomorphism $(\alpha, c)$ of $X_{1}=X(S, 1, F)$ onto $X_{2}=X\left(S^{\prime}, 1, F^{\prime}\right)$ with $\alpha S=S^{\prime}$ and $\alpha F=F^{\prime}+c$. Thus $\psi$ is of the form $\psi=\psi_{\alpha, c}$ $\in A\left(D_{p}\right)$. This observation proves the following lemma.

\section{Lemma 4.1. Let $X=X_{D_{p}, H}$. Then $\{\psi \in A(X) \mid \psi(1)=1\} \leqslant A\left(D_{p}\right)$.}

Let $X=X_{G, H}$. Since $\{\psi \in A(G) \mid \psi(H)=H\} \leqslant\{\psi \in A(X) \mid \psi(1)=1\}$ and that $X$ is a GRR of $G$ if and only if $\{\psi \in A(X) \mid \psi(1)=1\}=\{1\}$, we have

COROllary 4.2. $X_{D_{p}, H}$ is a GRR of $D_{p}$ if and only if there exists no nontrivial group automorphism $\psi \stackrel{\in}{\in} A\left(D_{p}\right)$ with $\psi(H)=H$.

TheOrem 4.3. Let $X=X(S, 1, F)$ where $0<|S| \leqslant(p-1) / 2$. Then $X$ is a $G R R$ of $D_{p}$ if and only if $F \notin \mathscr{F}$ for any $\mathscr{F}$ which is invariant under some nontrivial subgroup of $E(S)$.

Proof. If $F \notin \mathscr{F}$ for any $\mathscr{F}$ which is invariant under some nontrivial subgroup of $E(S)$, then $F \in \mathscr{F}$ for some $\mathscr{F} \in \mathscr{A}_{0}$. This means that $\alpha F \neq F+c$ for any $c \in Z_{p}$ unless $\alpha=1$ and $c=0$. By Corollary 4.2 and the above discussion, this implies that $X$ is a GRR of $D_{p}$.

Conversely let $X$ be a GRR of $D_{p}$. Since $|V(X)|=2 p, X \cong X_{D_{p}, H}, H=$ $\left\{a^{i}, a^{j} b \mid i \in S, j \in F\right\}$. If $F \in \mathscr{F}$ for some $\mathscr{F} \in \mathscr{A}_{i}(i \geqslant 1)$, then for some $1 \neq \alpha \in$ $E_{i} \subseteq E(S)$, we have $\alpha S=S$ and $\alpha F=F+c$ for some $c \in Z_{p}$. Since $X(S, 1, F)$ $\cong X(S, 1, F+d)$ for any $d \in Z_{p}$, we can assume without loss of generality that $F$ is such that $c=0$ so that $\alpha S=S$ and $\alpha F=F$. But then this $\alpha$ corresponds to a nontrivial group automorphism $\psi_{\alpha, 0}$ of $D_{p}$ such that $\psi_{\alpha, 0}(H)=H$. This however contradicts Corollary 4.2 that $X$ is a GRR of $D_{p}$.

Applying Theorem 4.3 and the results in Section 2, we obtain the following result. 
THEOREM 4.4. The number of $G R R X(S, 1, F)$ with $0<|S| \leqslant(p-1) / 2$ is given by

$$
s(p,|S|,|F|)=\sum_{d \text { even }} 2 I^{*}(p, d, 1,|F|) \beta^{*}(p,|S|, d)
$$

where the summation is extended over all even common divisors $d$ of $|S|$ and $p-1$.

We note that

$$
s(p, m, k)=s(p, m, p-k) \text { and } s(p, m, k)=s(p, p-m-1, p-k) \text {. }
$$

We remark that Theorem 4.3 is also true for $S=Z_{p}^{*}$. Now there is only one circulant graph $X(p, S)$ with $S=Z_{p}^{*}$ and hence

$$
s(p, p-1,|F|)=2 I^{*}(p, p-1,1,|F|)=\frac{1}{p-1} \sum_{d_{i}} \mu\left(d_{i}\right) \Psi\left(E_{i} ; F\right)
$$

where the summation is over all divisors $d_{i}$ of $p-1$ such that $d_{i}$ divides $|F|$ or $|F|-1$. Thus we have the following result.

THEOREM 4.5. $s(p, 0,|F|)=(1 /(p-1)) \sum_{d_{i}} \mu\left(d_{i}\right) \Psi\left(E_{i} ; F\right)$.

Note that if $|F| \leqslant 2$ or $|F| \geqslant p-2$, then $s(p, m,|F|)=0$ for any $m \geqslant 0$. We are interested in the case when $m=0$ and $|F|=3$. We shall omit the proof since it is straight forward.

Corollary 4.6. $s(p, 0,3)$ is equal to $(p-7) / 6$ if 3 divides $p-1$ and equal to $(p-5) / 6$ otherwise.

A group $G$ is said to have a cubic $G R R$ if there exists a GRR $X_{G, H}$ of $G$ with $|H|=3$.

Corollary 4.7. $D_{p}$ has a cubic GRR if and only if $p \geqslant 11$.

\section{References}

[1] B. Alspach, 'Point-symmetric graphs and digraphs of prime order and transitive permutation groups of prime degree,' J. Combin. Theory Ser. B. 15 (1973), 12-17.

[2] B. Alspach and R. Sutcliffe, 'Vertex-transitive graphs of order $2 p$, Ann. New York Acad. Sci. 319 (1979), 18-27.

[3] L. Babai, 'Isomorphism problem for a class of point symmetric structures,' Acta Math. Acad. Sci. Hungar. 29 (1977), 329-336. 
[4] G. L. Chia and C. K. Lim, 'A class of self-complementary vertex-transitive digraphs,' $J$. Combin. Theory, to appear.

[5] G. L. Chia, A class of strongly vertex transitive digraphs, (M. Sc. Thesis, University of Malaya, 1979).

[6] J. K. Doyle, 'Weak starred polygons and Cayley graphs,' Nanta Math. 11 (1978), 7-9.

[7] B. Elspas and J. Turner, 'Graphs with circulant adjecency matrices,' J. Combin. Theory 9 (1970), 297-307.

[8] C. D. Godsil, 'On Cayley graph isomorphisms,' Research Report No. 21, 1977, University of Melbourne.

[9] C. D. Godsil, 'Unsolved Problems,' Summer Research Workshop in Algebraic Combinatorics, July 1979, Simon Fraser University.

[10] F. Harary, Graph theory (Addison-Wesley, Reading, Menlo Park, London,1969).

[11] G. Sabidussi, 'On a class of fixed-point-free graphs,' Proc. Amer. Math. Soc. 9 (1958), 800-804.

[12] J. Turner, 'Point-symmetric graphs with a prime number of points,' J. Combin. Theory 3 (1967), $136-145$.

[13] M. E. Watkins, 'On the action of non-Abelian groups on graphs,' J. Combin. Theory 11 (1971), 95-104.

\section{Department of Mathematics}

University of Malaya

Kuala Lumpur 22-11

Malaysia 